\title{
Analysis of watermarking techniques in video
}

\begin{abstract}
Video piracy has become an increasing problem particularly with the proliferation of media sharing through the advancement of internet services and various storage technologies. Thus, research in copyright protection mechanisms, where one of which includes digital watermarking has been receiving an increasing interest from scientists especially in designing a seamless algorithm for effective implementation. Basically digital watermarking involves embedding secret symbols known as watermarks within video data which can be used later for copyright detection purposes. This paper presents the state of the art in video watermarking techniques. It provides a critical review on various available techniques. In addition, it addresses the main key performance indicators which include robustness, speed, capacity, fidelity, imperceptibility and computational complexity.
\end{abstract}

Keyword: Digital watermarking; Video watermarking; Watermarking 